\title{
Heartbeat: focus on heart disease in women
}

The relationship between hormone status and cardiovascular disease (CVD) in women remains controversial. CVD mortality is higher in women who experience period cessation at a younger age but few studies have examined the effect of menopause on risk factors for CVD. With ageing, there is an increased prevalence and severity of conventional CVD risk factors, including elevated serum lipid levels, hypertension, diabetes and body size, making it challenging to determine whether hormonal changes at menopause are an independent risk factor for CVD in women, particularly because previous studies have largely been based on a crosssectional study design. O'Keeffe and colleagues ${ }^{1}$ were able to examine the relationship between age at period cessation and trajectories of CVD risk factors using longitudinal data from 2547 women enrolled in the UK Medical Research Council National Survey of Health and Development, followed from birth in 1946 to the most recent visit at age 69 years. This data showed no association between age at period cessation (natural or surgical) and trajectories of serum lipid levels or blood pressure from age 36 to 69 years, with inconsistent associations for other CVD risk factors. This data suggests that the increased risk of adverse CVD outcomes with age in women is not mediated simply by changes in conventional CVD risk factors (figure 1).

In a compelling editorial, Gersh and Lavie $^{2}$ suggest that loss of normal ovarian function, specifically oestrogen production, is the likely mechanism of increased CVD risk after menopause, highlighting the many ways in which oestrogen might protect against CVD (figure 2). Further, they argue that 'The unintended legacy of the Women's Health Initiative (WHI), which tested the wrong "hormones" (not human-identical and given orally), on the wrong subjects (too old), must now finally be ended. The large body of scientific data that shows the harms of ovarian senescence and the CV benefits derived from hormones must now override the negative data derived from the WHI." They conclude: "More research is needed, but

Correspondence to Professor Catherine M Otto, Division of Cardiology, University of Washington, Seattle,WA 98195, USA; cmotto@uw.edu
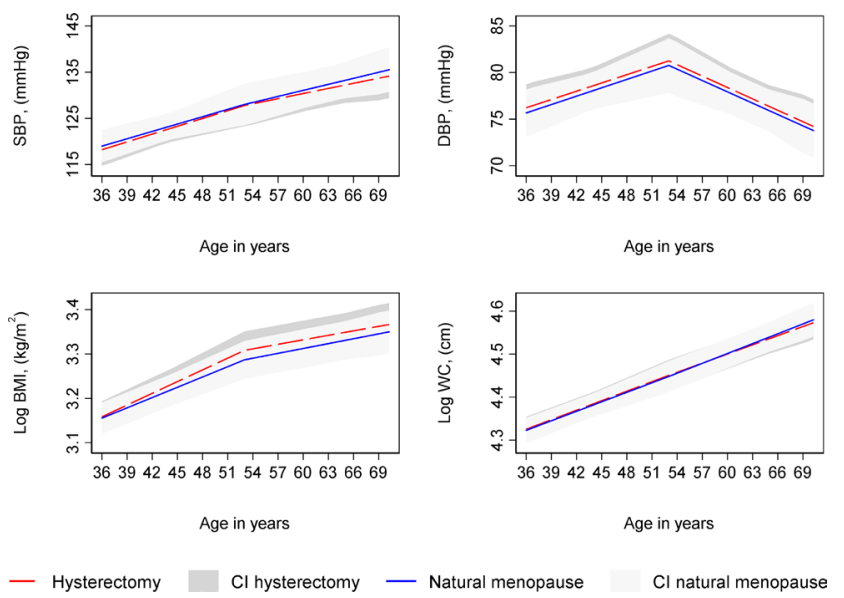

Figure 1 Mean predicted confounder adjusted trajectories of SBP, DBP, log BMI and log waist circumference from 36 to 69 years, by type of period cessation. Trajectories adjusted for socioeconomic position, age at period cessation, parity, time-varying hormone replacement therapy use, age at menarche, BMI at age 36 (SBP and DBP only), smoking at age 36, physical activity at age 36. BMI, body mass index; DBP, diastolic blood pressure; SBP, systolic blood pressure; wc, waist circumference.

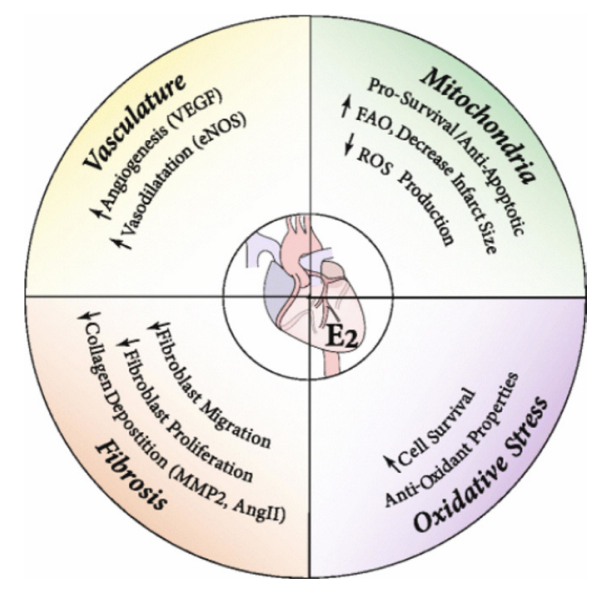

Figure 2 Summary of the likely protective mechanisms of oestrogen against cardiovascular disease. Illustration of the possible beneficial effects of oestrogen for the treatment of cardiovascular diseases such as ischaemic heart disease and heart failure. The protective effect of oestrogen in cardiovascular disease is associated with reduced fibrosis, stimulation of angiogenesis, and vasodilation, improved mitochondrial function and reduced oxidative stress. Angll, angiotensin II; eNOS, endothelial nitric oxide synthase; Fao, fatty acid oxidation; MMP2, matrix metalloproteinase 2; ROS, reactive oxygen species; VEGF, vascular endothelial growth factor. ${ }^{9}$ it is time now to acknowledge the impact of menopause - of ovarian senescence - on the cardiovascular health of women and treat them with physiological doses of human identical hormones, prior to the development of serious CVD sequela."

In another article in this issue of Heart, Ducas and colleagues ${ }^{3}$ performed a systemic review and meta-analysis of pregnancy outcomes in women with valvular heart disease (VHD), identifying 12 studies with a total of 646 pregnancies. There was a very high rate of adverse events in pregnant women with severe mitral stenosis (death in 3\%, pulmonary oedema in $37 \%$, preterm birth rate $18 \%$ ). Women with severe aortic stenosis also had high rates of adverse maternal and fetal outcomes (figure 3). Schmidt et $a l^{4}$ acknowledge the challenges in caring for pregnant women with VHD but hope this data will be a 'call for action' to improve prenatal counselling, evaluation and treatment of women with VHD and to ensure that those with moderate to severe VHD are referred to centres with expertise in their management.

Three original research papers in this issue of Heart address sex differences in cardiac arrhythmias. In patients with an implanted cardiac defibrillator (ICD) a long-detection time for arrhythmia 

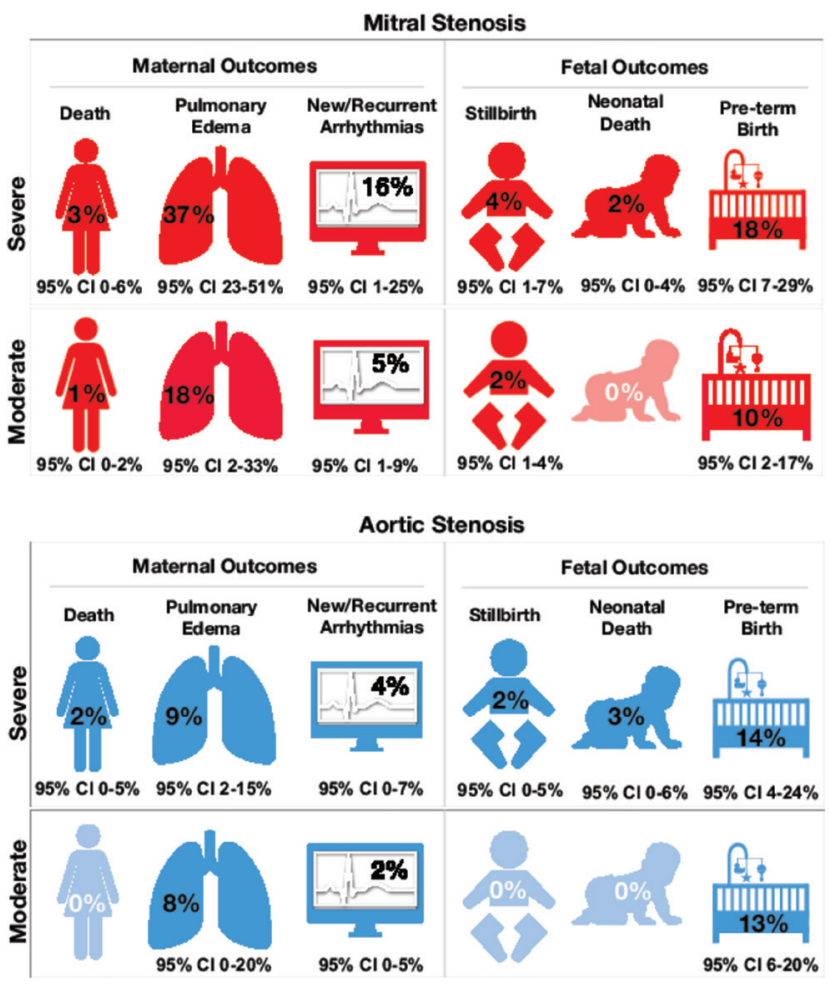

Figure 3 Maternal and fetal/neonatal outcomes by valve lesion and severity.

detection delivered fewer ICD therapies in women compared with men, suggesting that more arrhythmias self-terminated within 40 beats (compared with 24 beats with shorter detection times), with no sex difference in overall survival benefit of ICDs. ${ }^{5}$ In patients with atrial fibrillation

(AF), the risk of ischaemic stroke varied with age in women compared with men, being lower in women under age 66 yearsn, not different in women age 66-75 years, and higher only in those over age 75 years, suggesting that the conventional scores for estimated stroke risk in
AF patients might need to be modified. ${ }^{6}$ Women with heart failure and atrial fibrillation, are older, have different comorbidities fewer catheter ablations, and longer hospitalisations, although in-hospital mortality is similar compared with men after multivariable adjustment. ${ }^{7}$

A detailed review article by Mehilli and Presbitero ${ }^{8}$ discusses coronary artery disease and acute coronary syndrome in women, summarising the features specific to women in terms of risk factors, clinical presentations, diagnosis and findings on coronary angiography (figure 4).

\section{Contributors All authors contributed.}

Funding The authors have not declared a specific grant for this research from any funding agency in the public, commercial or not-for-profit sectors.

Competing interests None declared.

Patient consent for publication Not required.

Provenance and peer review Commissioned; internally peer reviewed.

(c) Author(s) (or their employer(s)) 2020. No commercial re-use. See rights and permissions. Published by BMJ.

\section{(A) Check for updates}

To cite Otto CM. Heart 2020;106:477-478.

Heart 2020;106:477-478.

doi:10.1136/heartjnl-2020-316802

\section{ORCID ID}

Catherine M Otto http://orcid.org/0000-0002-05279392

\section{REFERENCES}

1 O'Keeffe LM, Kuh D, Fraser A, et al. Age at period cessation and trajectories of cardiovascular risk factors across mid and later life. Heart 2020;106:499-505.

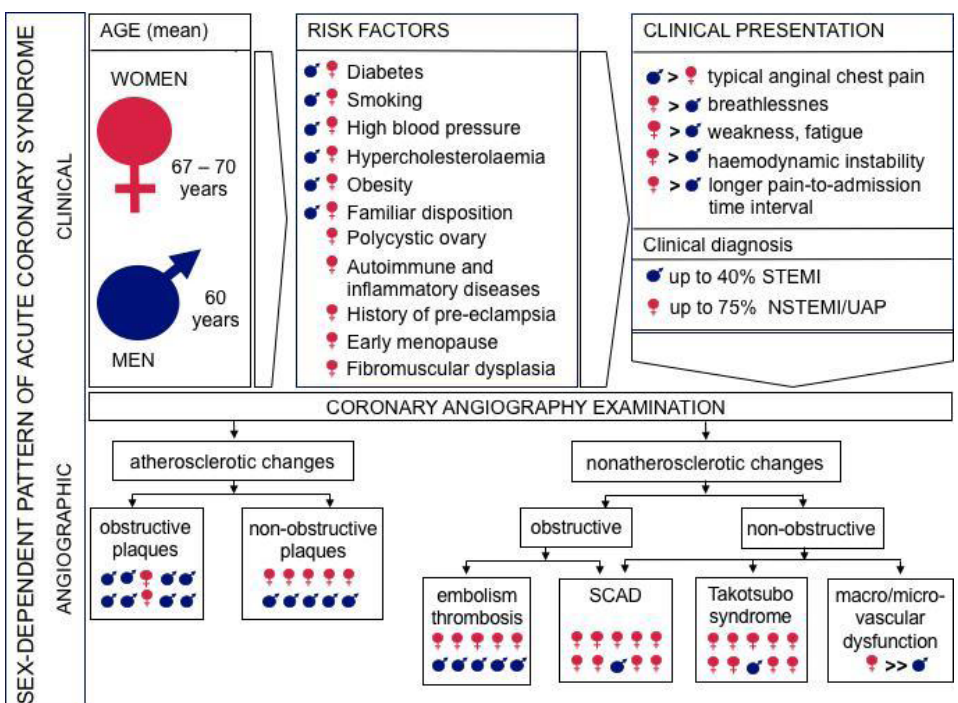

Figure 4 Sex-specific features of clinical and angiographic pattern of acute coronary syndrome. (N)STEMI, (non)ST-segment elevation myocardial infarction; SCAD, spontaneous coronary artery dissection.

2 Gersh FL, Lavie CJ. Menopause and hormone replacement therapy in the 21 st century. Heart 2020;106:479-81.

3 Ducas RA, Javier DA, D'Souza R, et al. Pregnancy outcomes in women with significant valve disease: a systematic review and meta-analysis. Heart 2020;106:512-9

4 Stefanescu Schmidt AC, Stetson B, Rigolin VH. Pregnancy in women with valvular heart disease: a call to action to reduce morbidity and mortality in both mother and child. Heart 2020;106:482-3.

5 Gasparini M, Kloppe A, Lunati M, et al. Sex differences in implantable cardiac defibrillator therapy according to arrhythmia detection times. Heart 2020;106:520-6

6 Wu VC-C, Wu M, Aboyans V, et al. Female sex as a risk factor for ischaemic stroke varies with age in patients with atrial fibrillation. Heart 2020;106:534-40.

7 Ueberham L, König S, Hohenstein S, et al. Sex differences of resource utilisation and outcomes in patients with atrial arrhythmias and heart failure. Heart 2020;106:527-33.

8 Mehilli J, Presbitero P. Coronary artery disease and acute coronary syndrome in women. Heart 2020;106:487-92.

9 lorga A, Cunningham CM, Moazeni S, et al. The protective role of estrogen and estrogen receptors in cardiovascular disease and the controversial use of estrogen therapy. Biol Sex Differ 2017;8:33. 